\title{
Spatiotemporal Normalization for Longitudinal Analysis of Gray Matter Atrophy in Frontotemporal Dementia
}

\author{
Brian Avants, Chivon Anderson, Murray Grossman, and James C. Gee \\ Depts. of Radiology and Neurology \\ University of Pennsylvania \\ Philadelphia, PA 19104-6389 \\ avants@grasp. cis . upenn. edu
}

\begin{abstract}
We present a unified method, based on symmetric diffeomorphisms, for studying longitudinal neurodegeneration. Our method first uses symmetric diffeomorphic normalization to find a spatiotemporal parameterization of an individual's image time series. The second step involves mapping a representative image or set of images from the time series into an optimal template space. The template mapping is then combined with the intrasubject spatiotemporal map to enable pairwise statistical tests to be performed on a population of normalized time series images. Here, we apply this longitudinal analysis protocol to study the gray matter atrophy patterns induced by frontotemporal dementia (FTD). We sample our normalized spatiotemporal maps at baseline (time zero) and time one year to generate an annualized atrophy map (AAM) that estimates the annual effect of FTD. This spatiotemporal normalization enables us to locate neuroanatomical regions that consistently undergo significant annual gray matter atrophy across the population. We found the majority of annual atrophy to occur in the frontal and temporal lobes in our population of 20 subjects. We also found significant effects in the hippocampus, insula and cingulate gyrus. Our novel results, significant at $p<0.05$ after false discovery rate correction, are represented in local template space but also assigned Talairach coordinates and Brodmann and Anatomical Automatic Labeling (AAL) labels. This paper shows the statistical power of symmetric diffeomorphic normalization for performing deformation-based studies of longitudinal atrophy.
\end{abstract}

\section{Introduction}

Neurodegeneration is a family of progressive disease processes affecting both the middle-aged and elderly. The economic impact of these diseases on families and society grows along with the size of the aging population. The relative number of at-risk individuals in the United States is projected to increase over the coming years. Developing biomarkers for early detection, assessment and treatment of this class of diseases is therefore of great significance.

Longitudinal studies of patient change are particularly valuable because of the difficulty in interpreting isolated observations in aging individuals. The most

N. Ayache, S. Ourselin, A. Maeder (Eds.): MICCAI 2007, Part II, LNCS 4792, pp. 303-310, 2007.

(C) Springer-Verlag Berlin Heidelberg 2007 
common method for following the demise of patients longitudinally is clinical assessment, such as monitoring performance during the repeated presentation of a cognitive task like confrontation naming or category naming fluency. Cognitive testing thus is able to use changing cognitive abilities to track disease progression. Because FTD can be difficult to monitor longitudinally with clinical measures, it is important to identify an objective method to support a clinical diagnosis. Jack 1 showed that segmentation-based, structure specific atrophy estimates derived from serial MRI are more statistically powerful than cognitive tests for tracking AD progress. In this study, we use a disease-specific optimal template and symmetric diffeomorphic normalization to examine FTD induced longitudinal atrophy at the full MRI resolution. FTD is an early-onset neurodegenerative condition with an average age of onset in the sixth decade of life. The conditions is as common as $\mathrm{AD}$ in individuals less than 65 years of age. The major clinical features of FTD include progressive aphasia or a disorder of social comportment and personality together with limited executive resources. Survival is typically eight years from onset. The disease is due to a disorder of tau metabolism or the accumulation of a ubiquinated protein known as TDP-43.

Patterns of longitudinal atrophy are increasingly investigated in a patient specific manner (a patient is used as his/her own control). A variety of methods is used for measuring brain change over time 2. Four quadrants defined in the brain were used to assess the global and regional atrophy rate in both FTD and Alzheimer's Disease (AD) 3]. Fox used deformable image registration to map the annual regional atrophy caused by AD [4] and to uncover presymptomatic changes in the medial temporal lobes. Scahill used statistical parametric mapping (SPM) 5] to detail AD atrophy [6, which was found to affect the hippocampus before clinical signs of AD appeared. Similar (but not symmetric) studies may also measure longitudinal change of specific structures [7]. Voxel compression mapping found early involvement of the posterior cingulate, temporoparietal cortex, and medial temporal lobes [4. Sowell [8] used surface-based methods to track pediatric brain development longitudinally. We focus on using our latest methodological advances to examine longitudinal FTD change in this study. The core contribution of this work is a strategy for using symmetric normalization to perform a template-based longitudinal analysis of whole brain gray matter atrophy. The integral and novel components of our method include: (1) a pure intrasubject measurement of longitudinal atrophy; (2) a spatiotemporal interpolation of the estimated annual change; and (3) leveraging the ability to compose diffeomorphic solutions to bring necessary variables into a common template space. To our knowledge, this is the first statement of a consistent, large deformation, image-based processing protocol for longitudinal studies of gray matter atrophy.

\section{Methods}

The goal of our processing is to use imaging data to estimate intrasubject annual change in cortical anatomy. We choose to use diffeomorphisms (differentiable 
and invertible maps with differentiable inverse) for this study due to their ability to capture both subtle intrasubject shape changes and the large deformations required when transforming a neurodegenerative brain into a normalized template space. Our diffeomorphisms are maps that take a position $\mathbf{x}$ in domain $\Omega$ back to $\Omega$. A path (or set) of such maps, parameterized by $t$, may be written $\phi: \mathbf{x} \in \Omega \times t \in[0,1] \rightarrow \Omega$ where, for each $t$ we have a unique diffeomorphism. Furthermore, a small change in $t$ gives a small change in the diffeomorphism as each new diffeomorphism is generated by integrating an ordinary differential equation $d \phi(\mathbf{x}, t) / d t=\boldsymbol{v}(\phi(\mathbf{x}, t), t)$. The $\boldsymbol{v}$ gives the tangent to the diffeomorphism at time $t$ and is a smooth vector field [9]. In image registration, the $\boldsymbol{v}$ at each time is determined by the minimization and regularization of the similarity term relating two images, $I$ and $J$.

We use a novel symmetric formulation for diffeomorphic image registration, referred to as symmetric normalization (SyN) [10], which does not require one to choose a "fixed" and "moving" image. Rather, both images deform smoothly along the shortest length path of diffeomorphisms connecting them. This property eliminates bias towards a specific, arbitrary reference frame and also enables features from both images to guide the mapping. Furthermore, SyN naturally includes a spatiotemporal parameterization of the image to image transformation allowing one to estimate transformations "in between" the end-points. These interpolated diffeomorphic transformations come from the large deformation analogy to a (small deformation) linear estimate of atrophy. This is because they are based on the notion of a shortest path in the space of diffeomorphisms.

We write the SyN energy as,

$$
\begin{array}{r}
E_{S y N}(I, J)=\inf _{\phi_{1}} \inf _{\phi_{2}} \int_{t=0}^{0.5}\left\{\left\|\boldsymbol{v}_{1}\right\|_{L}^{2}+\left\|\boldsymbol{v}_{2}\right\|_{L}^{2}\right\} d t \\
+\int_{\Omega} \omega\left|I\left(\phi_{1}(\mathbf{x}, 0.5)\right)-J\left(\phi_{2}(\mathbf{x}, 0.5)\right)\right|^{2} d \Omega . \\
\text { with each } \phi_{i} \text { the solution of: } \\
d \phi_{i}(\mathbf{x}, t) / d t=\boldsymbol{v}_{i}\left(\phi_{i}(x, t), t\right) \text { with } \phi_{i}(\mathbf{x}, 0)=\mathbf{x}
\end{array}
$$

where the value of $\omega$ weights the regularization versus the image matching term. Minimization with respect to $\phi_{1}$ and $\phi_{2}$, upholding a constant arc length constraint, provides an intrinsically symmetric image registration solution. The full map from $I$ to $J$ (or similarly from $J$ to $I$ ) is gained by composition, $\phi_{2}^{-1}\left(\phi_{1}(\mathrm{x}, 0.5), 0.5\right)$.

SyN builds on prior work on diffeomorphic representations [9] of image populations. SyN may also be used to symmetrically generate dataset-specific optimal local templates [11, known to improve localization accuracy and statistical significance 12 . These advances eliminate the template selection process and enable statistically fair comparisons while also guaranteeing the ability to capture the finest shape differences in one's data. We use a previously derived disease and scanner specific optimal template for the analyses in this manuscript. This template appears in figures 1 and 2 and was derived by iteratively optimizing equation 1 over a full image population, along with a parameter that controls 
for the template shape and appearance [11. The template includes three tissue segmentation, a cortical labeling and segmented deep cortical structures, all of which were prepared with semi-automated tools. Our study is restricted to the gray matter cortex. Similar (not symmetric) local template construction has been done in 13:14,15.

\section{Longitudinal Analysis via Intrasubject and Template Normalization:}

Our novel, symmetric diffeomorphic image registration tools permits us to deform the later time point structural MRI of each patient, referred to as the endpoint image, into the shape at the earlier time point, referred to as the baseline image, as in equation 1 . We refer to the output of this normalization, for image $i$, as $\phi^{i}(\mathbf{x}, t)$ where $\phi^{i}(\mathbf{x}, 0)$ maps to the baseline image and $\phi^{i}(\mathbf{x}, 1)$ maps to the last image in the time series. This physically based registration gives the structural change on a voxel-by-voxel basis, as shown in figure 1. The diffeomorphism is then sampled at time (with respect to the diffeomorphic parameterization $) t=(1$ year $) /($ imaging time interval in years $)$, where the time interval is greater than 1. In figure 1, the value for $t$ would be 0.25 as the imaging time interval is four years. This sampling provides a model of the annualized atrophy occurring in the patient cortex. The Jacobian of this transformation gives us a measure of annual atrophy throughout the individual brain space and is adjusted for the total intracranial volume of each individual. We refer to this image as the annualized atrophy map (AAM) and represent it in the baseline image space as $J_{A A M}^{i}$, for image $i$.

Subsequent to the estimate of longitudinal change, the annualized structural atrophy map must be warped into a template space where it may be evaluated statistically. We also achieve this goal with SyN by symmetrically and diffeomorphically mapping the baseline image into our local template space. The baseline to template mapping, for subject $i$, is referred to as $\phi^{i T}$. The mapping from any image in an individual time series to the template is then uniquely determined by $\phi^{i}\left(\phi^{i T}(\mathbf{x}, 1), t\right)$. We represent the Jacobian of $\phi^{i T}$ as $J^{i}$. We then have, for each individual, a spatially varying normalized representation of the relative neuroanatomical volumes at time zero, given by $J^{i}$, and time one year later, given by $J_{A A M}^{i T}=J^{i} \times J_{A A M}^{i}\left(\phi^{i T}\right)$. A paired T-test may then be performed over the dataset between the time zero, $\left\{J^{i}\right\}$, and time one year sets of images, $\left\{J_{A A M}^{i T}\right\}$ where $i$ ranges from 1 to $n$.

Our novel, composition-based, "intrasubject-first" approach eliminates inconsistencies that may occur when mapping both baseline and later time images directly to a template. Rather, we generate intrasubject structural measures by solving the much more clearly framed intrasubject correspondence problem. Consider assessing the longitudinal change by mapping directly to the template. In this case, the measure of longitudinal change is confounded by the difficulty of making the baseline to template and endpoint to template intersubject normalizations exactly consistent with each other. Our strategy prefers to generate a "pure" measure of longitudinal change from intrasubject data alone. Normalization to the template is then performed separately on the baseline images. We choose baseline images because the FTD disease process increases brain 
abnormalities over time, including the degree of hyper/hypointensity, atrophy and lesions present in the brain. Furthermore, we represent the volume change from baseline to a later time in the baseline reference frame.

Subjects and Image Acquisition. We applied our methods to 20 patients diagnosed with an FTD spectrum disorder at the Department of Neurology at the University of Pennsylvania. Initial clinical diagnosis was established by an experienced neurologist (M.G.) using published criteria. Subsequently, at least two trained reviewers of a consensus committee confirmed the presence of specific diagnostic criteria based on an independent review of the semi-structured history, mental status examination and neurological examination. All subjects' images were acquired with a Siemens 3.0 T MRI scanner. Each study began with a rapid sagittal T1-weighted scan to determine patient position. A T1 structural acquisition was then acquired with TR (repetition time) $=1620 \mathrm{~ms}$, TE (echo time $)=3 \mathrm{~s}$, slice thickness: $1 \mathrm{~mm}$, in-plane resolution: $.9766 \mathrm{~mm} \times .9766 \mathrm{~mm}$ and matrix $256 \times 256 \times 192$. The same protocol was used at baseline and later time points.

\section{Results and Discussion}

We now present the results of our novel spatiotemporal approach to analyzing longitudinal atrophy via symmetric normalization. We evaluated the statistical significance of the difference between baseline and time one log-Jacobian images with the statistical parametric mapping software, SPM2 [5]. We used explicit masking to restrict the analysis to the gray matter cortex and used pairwise $\mathrm{T}$ tests to uncover significant regions of atrophy. Regions that undergo significant annual atrophy, due to the FTD effect on gray matter, are overlaid on our rendered template in figure 2] Details of our results, including Brodmann areas and AAL labels, are in table 11. Significance is defined as a minimum cluster size of 50 and false discovery rate corrected p-value $<0.05$. Note that we found no significant correlations between atrophy rate and global brain atrophy, nor between age and atrophy rate.

The significantly affected areas are located primarily in the temporal and frontal lobes, preferentially on the left. Affected subcortical regions include the left hippocampus, left insula and the cingulum bilaterally. The most significant atrophic effects were found in the left and right cingulum, left insula, left temporal pole and left hippocampus. Atrophy in these regions is also present on the right, but with less significance. The average annual atrophy over significant voxels was approximated as $6.5 \%$ and over all cortical gray matter as $3.3 \%$. Note that we also provide Brodmann and AAL labels at the most significant location in each cluster. We highlight that AAL and Brodmann labels do not necessarily agree, as there is some inconsistency in the coordinate systems used by each protocol.

Many of the indicated structures are involved in the cognitive resources used to monitor patients longitudinally. Cortical loss in these structures would sensibly affect cognitive performance, such as the ability to name animals or recall 

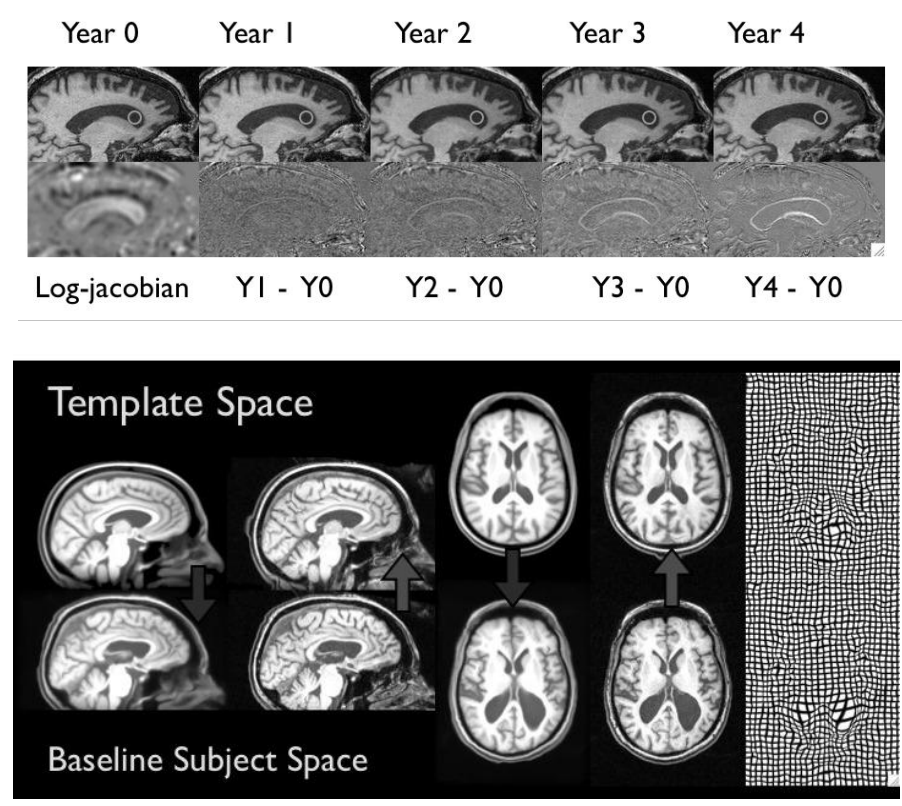

Fig. 1. We parameterize a single subject's temporal series with symmetric diffeomorphisms (top). This longitudinal transformation is the basis for our estimate of the annual atrophy map. The panel below shows the successful normalization of the baseline image for a single-subject to the template image, as well as the inverse mapping of the template to the baseline image. This mapping is used to normalize the full intrasubject image time series and the associated longitudinal transformations to the template. Normalizing the population of longitudinal transformations to the template space enables a paired T-test for significant atrophy between any two time points in the series.

the appropriate names for objects. In fact, patients with FTD are known to have difficulties with cognitive tasks like naming because of disease in the temporal lobe and executive functioning because of frontal lobe disease [16]. In particular, the left temporal lobe is compromised in semantic dementia, one presentation of FTD, and this is related to profound naming difficulty. The left insula and inferior frontal region is related to progressive non-fluent aphasia, another FTD presentation that involves effortful speech and reduced language fluency. Bilateral frontal regions such as the cingulum and orbital frontal cortex are most involved in the social and executive disorder associated with FTD [16. The results of this study suggest that structural MRI, combined with sensitive symmetric normalization techniques, has the ability to act as a reliable biomarker for patient studies of progressive neurodegeneration. Our future work will investigate the effect of FTD on the cognitive network and the relationship of atrophy in this network to loss of cognition. We also hope to gain further insight into the relationship between brain and behavior. Finally, we are currently evaluating our SyN-based structural measurements with respect to measures of annual atrophy gained by cortical thickness. 


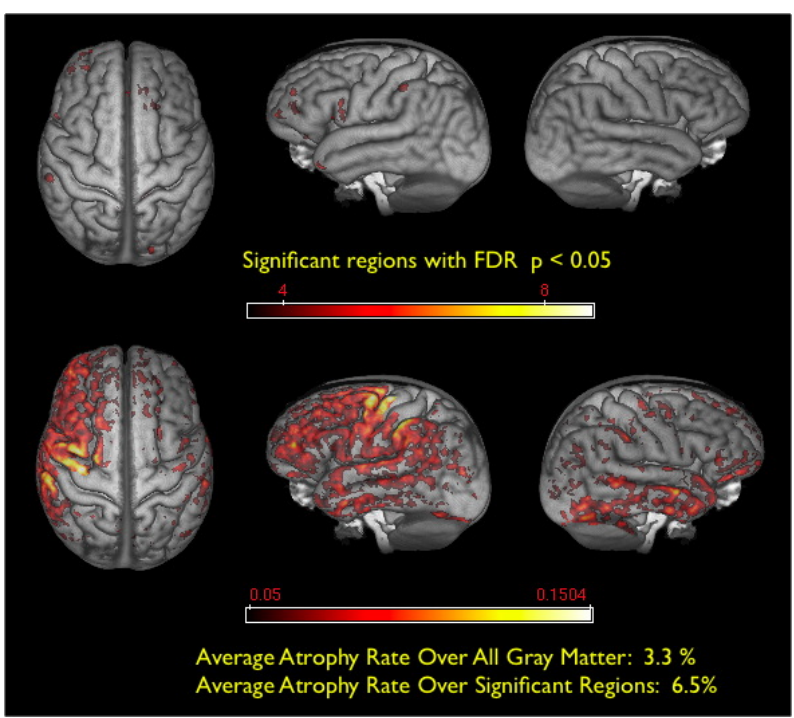

Fig. 2. Average annual FTD atrophy rate greater than or equal to $5 \%$ is shown at bottom, along with the associated atrophy rate scale (brighter means greater atrophy rate). The $p<0.05$ FDR corrected significant regions are shown at top, along with a brightness scale that reflects the local T-statistic value. Overall, the effect of atrophy, in this dataset, is more apparent on the left. Atrophy is most bilaterally present in the temporal lobes and cingula.

Table 1. Significantly Atrophic Regions with False Discovery Rate corrected $p<0.05$

\begin{tabular}{|l|c|c|c|c|c|}
\hline Cluster & max T & Clust Size & MNI Coord & Brodmann & AAL Label \\
\hline \hline 1 & 8.71 & 5171 & $11-2841$ & 23 & Cingulum Mid R \\
\hline 2 & 7.28 & 2499 & $-3-2536$ & 23 & Cingulum Mid L \\
\hline 3 & 5.83 & 101 & -29183 & - & Insula L \\
\hline 4 & 5.73 & 278 & -7317 & 25 & Cingulum Ant L \\
\hline 5 & 5.36 & 69 & $-3313-39$ & 20 & Temporal Pole Mid L \\
\hline 6 & 5.25 & 110 & $-15-6-16$ & 28 & Hippocampus L \\
\hline 7 & 5.19 & 79 & $-55-2444$ & 3 & Parietal Inf L \\
\hline 8 & 5.17 & 77 & -315112 & 10 & Frontal Mid L \\
\hline 9 & 5.02 & 110 & -561218 & 44 & Frontal Inf Oper L \\
\hline 10 & 4.84 & 98 & $1133-16$ & 11 & Frontal Mid Orb R \\
\hline 11 & 4.83 & 154 & $25-3-10$ & 34 & Amygdala R \\
\hline 12 & 4.77 & 106 & $260-24$ & 34 & Amygdala R \\
\hline 13 & 4.50 & 113 & $-2038-13$ & 11 & Frontal Lat Orb L \\
\hline 14 & 4.36 & 61 & $-4345-1$ & 46 & Frontal Mid Orb L \\
\hline
\end{tabular}




\section{References}

1. Jack, C.R., Shiung, M.M., Gunter, J.L., O'Brien, P.C., Weigand, S.D., Knopman, D.S., Boeve, B.F., Ivnik, R.J., Smith, G.E., Cha, R.H., Tangalos, E.G., Petersen, R.C.: Comparison of different mri brain atrophy rate measures with clinical disease progression in ad. Neurology 62(4), 591-600 (2004)

2. Cardenas, V.A., et al.: Comparison of methods for measuring longitudinal brain change in cognitive impairment and dementia. Neurobiology of Aging 24(4), 537$554(2003)$

3. Chan, D., Fox, N.C., Jenkins, R., Scahill, R.I., Crum, W.R., Rossor, M.N.: Rates of global and regional cerebral atrophy in $\mathrm{AD}$ and frontotemporal dementia. Neurology 57, 1756-1763 (2001)

4. Fox, N., Crum, W., Scahill, R., Stevens, J., Janssen, J., Rossor, M.: Imaging of onset and progression of alzheimer's disease with voxel-compression mapping of serial magnetic resonance images. Lancet 358, 201-205 (2001)

5. Ashburner, J., Hutton, C., Frackowiak, R., Price, C., Johnsrude, I., Friston, K.: Identifying global anatomical differences: Deformation-based morphometry. Hum. Brain Mapp. 6, 348-357 (1998)

6. Scahill, R.I., Schott, J., Stevens, J.: Mapping the evolution of regional atrophy in alzheimer's disease: unbiased analysis of fluid-registered serial mri. Proc. Natl. Acad. Sci. 99, 4135-4137 (2002)

7. van de Pol, L.A., Barnes, J., Scahill, R.I., Frost, C., Lewis, E.B., Boyes, R.G., van Schijndel, R.A., Scheltens, P., Fox, N.C., Barkhof, F.: Improved reliability of hippocampal atrophy rate measurement in mild cognitive impairment using fluid registration. Neuroimage 34(3), 1036-1041 (2007)

8. Sowell, E.R., Thompson, P.M., Leonard, C.M., Welcome, S.E., Kan, E., Toga, A.W.: Longitudinal Mapping of Cortical Thickness and Brain Growth in Normal Children. J. Neurosci. 24, 8223-8231 (2004)

9. Miller, M., Trouv'e, A., Younes, L.: On the metrics and Euler-Lagrange equations of computational anatomy. Annu. Rev. Biomed. Eng. 4, 375-405 (2002)

10. Avants, B., Grossman, M., Gee, J.C.: Symmetric diffeomorphic image registration: Evaluating automated labeling of elderly and neurodegenerative cortex. Medical Image Analysis (in press, 2007)

11. Avants, B., Gee, J.C.: Geodesic estimation for large deformation anatomical shape and intensity averaging. Neuroimage (Suppl. 1), S139-150 (2004)

12. Senjem, M., Gunter, J.L., Shiung, M.M., Petersen, R., Jack, C.R.: Comparison of different methodological implementations of voxel-based morphometry in neurodegenerative disease. Neuroimage 26(2), 600-608 (2005)

13. Lorenzen, P., Prastawa, M., Davis, B., Gerig, G., Bullitt, E., Joshi, S.: Multi-modal image set registration and atlas formation. Medical Image Analysis 19(3), 440-451 (2006)

14. Beg, M.F., Khan, A.: Computing an average anatomical atlas using LDDMM and geodesic shooting. In: ISBI, pp. 1116-1119 (2006)

15. Twining, C.J., Cootes, T., Marsland, S., Petrovic, V., Schestowitz, R., Taylor, C.J.: A unified information-theoretic approach to groupwise non-rigid registration and model building. In: Christensen, G.E., Sonka, M. (eds.) IPMI 2005. LNCS, vol. 3565, pp. 1-14. Springer, Heidelberg (2005)

16. Grossman, M., et al.: What's in a name?: voxel-based morphometric analyses of MRI and naming difficulty in alzheimer's disease, frontotemporal dementa and corticobasal degeneration. Brain 127(3), 628-649 (2004) 